\title{
Un Programa Breve Basado en la Solución de Problemas para la Prevención de la Depresión en Cuidadores Informales de Pacientes con Demencia: Un Estudio Piloto
}

\section{A Short Problem-solving Based Course for Prevention of Depression in Non-professional Providers of Care for Dementia Patients: A Pilot Study}

\author{
Fernando L. Vázquez, Patricia Otero, María López, Vanessa Blanco y Ángela Torres \\ Universidad de Santiago de Compostela
}

\begin{abstract}
Resumen. Se evaluó la utilidad potencial de aplicar un programa de prevención de la depresión para cuidadores con síntomas significativos de depresión en una muestra de 10 mujeres y 3 hombres de edades comprendidas entre los 43 y los 82 años $(M=59.9$ años; $D T=11.9)$. El programa constó de cinco sesiones grupales. Las evaluaciones tras la intervención mostraron una reducción estadísticamente significativa en la sintomatología depresiva $(p<.001)$, la inadaptación social $(p<.001)$, los niveles de sobrecarga $(p<.05)$, el riesgo de depresión y un incremento significativo en las habilidades de solución de problemas $(p<.001)$. El riesgo de depresión permaneció más bajo que en los niveles preintervención en el seguimiento realizado 12 semanas después de la intervención $(p<.01)$. Estos datos alentadores justifican una evaluación adicional del programa utilizando una muestra mayor.
\end{abstract}

Palabras clave: cuidador, demencia, prevención, depresión, solución de problemas.

\begin{abstract}
The potential utility of applying a programme for prevention of depression for care providers with significant symptoms of depression was evaluated using a sample of 10 women and 3 men aged $43-82$ years $(M=59.9$ years; $S D=11.9)$. The programme consisted of five group sessions. Post-intervention evaluations showed a statistically significant reduction in depressive symptoms $(p<.001)$, social maladjustment $(p<.001)$, burden levels $(p<.05)$, risk of depression $(p<.05)$, and a significant increase in problem-solving skills $(p$ $<.001)$. Risk of depression remained lower than pre-intervention levels at follow-up, performed 12 weeks after the intervention $(p<.01)$. These encouraging results justify further evaluation of the programme using a larger sample.

Keywords: care providers, dementia, prevention, depression, problem-solving.
\end{abstract}

La correspondencia sobre este artículo dirigirla al primer autor al Departamento de Psicología Clínica y Psicobiología. Facultad de Psicología. Universidad de Santiago de Compostela. Campus Sur. 15782 Santiago de Compostela. España. E-mail: fernandolino.vazquez@usc.es.
Agradecimientos: Este trabajo ha sido financiado por el Proyecto 2007/PN018 del Ministerio de Trabajo y Asuntos Sociales. También ha contado con el apoyo de la Dirección General de Ordenación y Calidad del Sistema Universitario de Galicia, de la Consellería de Educación y Ordenación Universitaria-Xunta de Galicia. Asimismo, los autores agradecen la ayuda prestada por la Asociación de Familiares de Alzheimer de Coruña (AFACO) y la Asociación de Familiares de Enfermos de Alzheimer de Santiago de Compostela (AGADEA). 


\section{Introducción}

La depresión es considerada frecuentemente como el "resfriado" de los trastornos mentales (Dozois y Dobson, 2004). Aunque esta afirmación es acertada en términos de su elevada prevalencia, tiende a minimizar las consecuencias de la depresión a nivel personal y social. Según un informe de la Organización Mundial de la Salud (OMS), la depresión representa la principal causa de discapacidad en Europa, y constituye la patología psíquica más prevalente a nivel mundial, afectando aproximadamente a 340 millones de personas (OMS, 2004).

Una de las poblaciones con mayores porcentajes de depresión son los cuidadores informales de personas dependientes. Entre otros hallazgos, se ha encontrado que entre el $40 \%$ y el $70 \%$ de los cuidadores tienen niveles clínicos significativos de síntomas depresivos y en torno al $50 \%$ podrían reunir los criterios diagnósticos para un trastorno depresivo mayor (Gallagher, Rose, Rivera, Lovett y Thompson, 1989; Maidment, Regan, Katona y Livingston, 2005; Schulz, O'Brien, Bookwala y Fleissner, 1995). Algunos factores de riesgo encontrados para la depresión en esta población son, entre otros, la propia condición de familiar de la persona que cuida, los problemas de conducta, el deterioro cognitivo y la dependencia funcional en las actividades de la vida diaria del paciente, el número de horas y la cantidad de tiempo dedicados al cuidado, el bajo apoyo social y la sobrecarga (Covinsky et al., 2003; Moral Serrano, Ortega, López Matoses y Pellicer Magraner, 2003). En los cuidadores con elevados niveles de sintomatología depresiva se ha encontrado una peor calidad de vida (Vitaliano, Young y Zhang, 2004), y un elevado riesgo de mortalidad comparado con sujetos control que no actúan como cuidadores (Schulz y Beach, 1999). Es más, un deterioro en la salud física y mental del cuidador puede influir negativamente en la persona que necesita sus cuidados, estando asociado con la supervivencia del paciente (McClendon, Smyth y Neundorfer, 2004) y el tiempo de institucionalización (Gilley, McCann, Bienias y Evans, 2005).

Ante la evidencia de estos elevados niveles de depresión en los cuidadores son necesarias intervenciones que los apoyen tanto emocionalmente como en la realización de sus actividades de cuidado. En las últimas dos décadas se han ido desarrollando distintos programas de intervención psicológica para cuidadores, especialmente para aquellos que cuidan enfermos con demencia (ej., Eisdofer et al., 2003; Lovett y Gallagher, 1988; Teri, Logsdon, Uomoto y McCurry, 1997). Estos programas han utilizado diferentes formatos y métodos para ayudar a los cuidadores, comprendiendo intervenciones psicológicas individuales o en grupo, programas psicoeducativos, grupos de apoyo, intervenciones familiares, programas multimodales, intervenciones basadas en tecnologías, etc. La valoración de los mismos en metaanálisis recientes sugiere que las intervenciones para cuidadores en su conjunto son efectivas, pero sus efectos son relativamente pequeños y en áreas específicas (Brodaty, Green y Koschera, 2003; López y Crespo, 2007; Pinquart y Sörensen, 2006; Sörensen, Duberstein, Gill y Pinquart, 2006).

Además, sólo un pequeño número de estudios han evaluado como resultado principal la depresión (Coon, Thompson, Steffen, Sorocco y GallagherThompson, 2003; Mittelman, Roth, Coon y Haley, 2004). La razón fundamental de ello es que la gran mayoría de las investigaciones se han centrado en dotar a los cuidadores de conocimientos, estrategias y habilidades que les ayuden a sobrellevar su labor como cuidador (Gallagher-Thompson et al., 2000; Roberts et al., 1999), y no tanto en tratar su problemática individual o los trastornos específicos que pudieran sufrir. Asimismo, ninguna de esas intervenciones realizadas con cuidadores se han centrado únicamente en cuidadores con alto riesgo de desarrollar una depresión en los que se hayan identificado manifestaciones prodrómicas o subclínicas, pero en los que todavía no se cumplen los criterios para este trastorno. De igual modo, ningún estudio ha realizado un programa de prevención de la depresión, pese al problema de infradiagnóstico de este trastorno y su morbilidad oculta (Carrillo, Rojo y Staats, 2004), a las recomendaciones de llevar a cabo intervenciones de tipo preventivo que puedan evitar los elevados costes personales, sociales y económicos que conlleva la depresión (OMS, 2004; Vázquez y Torres, 2007), y al riesgo elevado de la aparición de episodios depresivos en la población de cuidadores informales de las personas dependientes. 
Es más, la mayor parte de estos estudios realizados con cuidadores informales de personas con demencia no parten de un modelo teórico en el que apoyar el programa de intervención propuesto. En este sentido, López y Crespo (2007) y Montorio, Yanguas y Díaz-Veiga (1999) han señalado la necesidad de definir con claridad el modelo teórico que subyace al diseño de los programas de intervención con cuidadores con el fin de posibilitar la valoración de la eficacia de distintas formas de intervención. La consecuencia de esta situación en la actualidad es la carencia de estudios en los que se evalúen los mediadores de los efectos de la intervención (Pinquart y Sörensen, 2006), llevando al desconocimiento de los factores que explican la eficacia de los tratamientos.

Asimismo, cuando se han diseñado la mayoría de estas intervenciones no se ha tenido en cuenta su potencial aplicabilidad en los contextos en los que los cuidadores demandan ayuda. Son necesarios programas de intervención "contextualizados" que se adapten a las necesidades específicas de los cuidadores (López y Crespo, 2007), que alcancen los objetivos del tratamiento en el menor número de sesiones posibles, que sean eficaces, que sean accesibles en términos de tiempo y dinero, que sean atractivos para los clínicos (breves, fáciles de aprender y con implicaciones específicas para la práctica clínica) y para los cuidadores (Sörensen et al., 2006) y que se puedan aplicar en los contextos en los que los cuidadores solicitan ayuda. El número de sesiones invertidas en las intervenciones realizadas hasta la actualidad es variable, pero la mayoría se sitúan en torno a 8-10 sesiones (Chang, 1999; Coon et al., 2003), y son escasas las intervenciones breves. Sólo en un estudio (Schmidt, Bonjean, Widem, Schefft y Steele, 1988) se realizó una intervención inferior a cinco sesiones. Sin embargo, en éste no se planteó un programa de intervención sistematizado.

El objetivo de este estudio piloto es evaluar la viabilidad de un programa de intervención breve basado en la terapia de solución de problemas para prevenir la depresión en los cuidadores informales de personas con demencia que presenten sintomatología depresiva sin llegar a cumplir los criterios diagnósticos para el trastorno de depresión mayor (prevención indicada). Se espera que, tras la aplica- ción de la intervención, tenga lugar un cambio en las puntuaciones de sintomatología depresiva, de habilidades de solución de problemas, de ajuste social y de sobrecarga experimentada por los cuidadores.

\section{Método}

\section{Sujetos}

En este estudio se empleó un diseño pretest-postest sin grupo de comparación con una medida de seguimiento a los tres meses. Los participantes eran cuidadores informales, esto es, personas encargadas del cuidado no remunerado y no vinculado a un servicio de atención profesional prestado normalmente por un familiar a un sujeto que requiere ayuda en la ejecución de las actividades básicas de la vida diaria (Mossialos, Allin y Figueras, 2007; Rivera, 2001), con independencia de si convivían o no con la persona receptora de los cuidados. Dichos participantes se reclutaron durante los meses de abril, mayo y septiembre de 2007 en dos asociaciones federadas de familiares de enfermos de Alzheimer de Galicia.

Para participar en el estudio cada sujeto tenía que a) ser cuidador informal de un familiar en situación de dependencia por sufrir la enfermedad de Alzheimer o algún otro tipo de demencia (diagnosticada anteriormente por un médico), b) pertenecer a una asociación federada de Galicia relacionada con la enfermedad de Alzheimer u otras demencias, c) someterse a una evaluación psicológica realizada por un psicólogo mediante una entrevista y la cumplimentación de cuestionarios, d) encontrarse en una situación de riesgo de depresión determinada por una puntuación en la Escala para la Depresión del Centro de Estudios Epidemiológicos (CES-D; Radloff, 1977) igual o mayor al punto de corte $(\geq 16)$, e) estar de acuerdo en participar en la evaluación de seguimiento, y f) firmar el consentimiento informado de participación en el tratamiento. Se excluyeron a aquellos sujetos que en el momento de la evaluación a) experimentaran un episodio depresivo mayor, b) estuvieran recibiendo un tratamiento psicológico o farmacológico, c) presentasen otros trastornos mentales o médicos que pudieran actuar como variables de confusión en el estudio (ej., sín- 
tomas debidos a los efectos fisiológicos directos de una sustancia o una condición médica), o d) presentasen trastornos mentales o médicos que por su gravedad requiriesen una inmediata intervención (ej., ideación suicida).

Los sujetos fueron contactados personalmente, informados de la naturaleza del estudio e invitados a participar, contestando a todas las dudas y cuestiones que plantearon. A todos ellos se les garantizó la confidencialidad y el anonimato. La participación fue completamente voluntaria, sin ninguna clase de incentivo económico o de otro tipo.

Como se puede observar en la Tabla 1, la muestra final estuvo formada por 13 sujetos $(76.9 \%$ mujeres), con un rango de edad comprendido entre los 43 y los 82 años $(M=59.9$ años; $D T=11.9)$.

Inicialmente, se evaluaron un total de 21 sujetos, de los cuales tres fueron eliminados por no cumplir los criterios de inclusión. Tres de los 18 sujetos elegibles declinaron participar, y dos decidieron abandonar el tratamiento durante la fase de intervención o la fase de seguimiento. Las razones referidas para los rechazos y abandonos fueron problemas de salud $\mathrm{y}$ falta de tiempo para implicarse en el estudio.

\section{Instrumentos}

Cuestionario de información sobre características de los cuidadores y familiares con demencia. Se recogieron datos sobre variables sociodemográficas y clínicas del cuidador y de la persona cuidada (ver Tabla 1).

La Escala para la Depresión del Centro de Estudios Epidemiológicos (CES-D; Radloff, 1977; versión española de Vázquez, Blanco y López, 2007). Es una escala autoinformada de 20 ítems que proporciona una puntuación continua sobre el nivel de sintomatología depresiva durante la última semana. Cada uno de los 20 ítems es evaluado en una escala tipo Likert de cuatro opciones de respuesta que van desde el 0 (raramente o ninguna vez) al 3 (la mayor parte del tiempo). El rango de las puntuaciones abarca de 0 a 60 , donde una mayor puntuación corresponde a una mayor sintomatología depresiva. Un punto de corte de 16 o mayor es indicativo de alto riesgo para desarrollar una depresión clínica.
Este cuestionario muestra una consistencia interna relativamente alta $(\alpha=.87)$ (Garand, Dew, Eazor, DeKoski y Reynolds, 2005), y una adecuada validez predictiva para el diagnóstico de depresión (Bergman-Evans, 1994). La adaptación al castellano (Vázquez et al., 2007) también ha mostrado una alta consistencia interna $(\alpha=.89)$.

Entrevista Clínica Estructurada para los Trastornos del Eje I del DSM-IV, Versión Clínica (SCID-CV; First, Spitzer, Gibbon y Williams, 1997/1999). Es una entrevista semiestructurada que proporciona diagnósticos del DSM-IV (American Psychiatric Association; APA, 1995) y debe ser administrada por un clínico. Está compuesta por seis módulos (episodios afectivos, síntomas psicóticos, trastornos psicóticos, trastornos por uso de substancias, trastornos de ansiedad y otros trastornos) de entrevista diagnóstica. De la totalidad de la entrevista, sólo se ha utilizado el módulo correspondiente a los trastornos depresivos. En cuanto a sus propiedades psicométricas, muestra una buena fiabilidad testretest y una alta fiabilidad para pacientes psiquiátri$\cos$.

El Inventario de Solución de Problemas Revisado (Social Problem-Solving Inventory-Revised; SPSI$R$, D’Zurilla, Nezu, y Maydeu-Olivares, 1997; versión española de Maydeu-Olivares, RodríguezFornells, Gómez-Benito y D'Zurilla, 2000). Es un cuestionario autoaplicable que evalúa las habilidades para resolver los problemas. Consta de 52 ítems en una escala tipo Likert con varias opciones de respuesta que van desde el 0 (no es típico en mî) al 4 (extremadamente típico en mí). Proporciona una puntuación total y nueve dimensiones: orientación positiva hacia el problema, orientación negativa hacia el problema, estilo impulsivo, estilo evitativo, solución de problemas racional, definición y formulación del problema, generación de soluciones alternativas, toma de decisiones e implementación y verificación de la solución. Unas puntuaciones elevadas son indicativas de unas adecuadas habilidades de solución de problemas, excepto en orientación negativa hacia el problema, estilo impulsivo y estilo evitativo, donde unas puntuaciones altas indican déficits en estas habilidades. La consistencia interna se sitúa entre 0.69 y 0.95 , la correlación test-retest entre 0.72 y 0.88 y ha demostrado tener validez 
Tabla 1.Características de los cuidadores y familiares con demencia que completaron el estudio $(N=13)$

\begin{tabular}{|c|c|c|}
\hline Cuidadores & $N$ & $\%$ \\
\hline \multicolumn{3}{|l|}{ Edad (años) } \\
\hline$M$ & 59.9 & \\
\hline$D T$ & 11.9 & \\
\hline \multicolumn{3}{|l|}{ Sexo } \\
\hline Mujer & 10 & 76.9 \\
\hline Hombre & 3 & 23.1 \\
\hline \multicolumn{3}{|l|}{ Estado civil } \\
\hline Soltero/a & 3 & 23.1 \\
\hline Casado/a & 10 & 76.9 \\
\hline \multicolumn{3}{|l|}{ Clase social } \\
\hline Baja / Media-baja & 2 & 15.4 \\
\hline Media & 9 & 69.2 \\
\hline Media-alta / Alta & 2 & 15.4 \\
\hline \multicolumn{3}{|l|}{ Ingresos mensuales por unidad familiar } \\
\hline Hasta $999 €$ & 1 & 7.7 \\
\hline De 1000 a $1499 €$ & 4 & 30.8 \\
\hline De 1500 a $1999 €$ & 2 & 15.4 \\
\hline De $2000 €$ en adelante & 5 & 38.5 \\
\hline No sabe / No contesta & 1 & 7.7 \\
\hline \multicolumn{3}{|l|}{ Nivel de estudios } \\
\hline Sin estudios (pero sabe leer y escribir) & 1 & 7.7 \\
\hline Primarios & 3 & 23.1 \\
\hline Secundarios & 8 & 61.5 \\
\hline Universitarios & 1 & 7.7 \\
\hline \multicolumn{3}{|l|}{ Situación laboral } \\
\hline Ocupado & 4 & 30.8 \\
\hline No ocupado & 9 & 69.2 \\
\hline Jubilado & 3 & 23.1 \\
\hline Tareas del hogar & 6 & 46.1 \\
\hline \multicolumn{3}{|l|}{ Parentesco con la persona cuidada } \\
\hline Cónyuge & 4 & 30.8 \\
\hline Hijo/a & 8 & 61.5 \\
\hline Otros & 1 & 7.7 \\
\hline \multicolumn{3}{|l|}{ Tiempo cuidando (años) } \\
\hline$M$ & 5.6 & \\
\hline$D T$ & 5.1 & \\
\hline \multicolumn{3}{|l|}{ Horas diarias dedicadas al cuidado } \\
\hline M & 13.8 & \\
\hline$D T$ & 6.3 & \\
\hline \multicolumn{3}{|l|}{ Vive con la persona a la que cuida } \\
\hline Sí & 12 & 92.3 \\
\hline No & 1 & 7.7 \\
\hline \multicolumn{3}{|l|}{ Puntuación CES-D } \\
\hline$M$ & 26.3 & \\
\hline \multirow{2}{*}{\multicolumn{3}{|c|}{ Puntuación SPSI-R }} \\
\hline & & \\
\hline$M$ & 85 & \\
\hline$D T$ & 12.3 & \\
\hline \multicolumn{3}{|l|}{ Puntuación SAS-M } \\
\hline M & 2.4 & \\
\hline$D T$ & 0.4 & \\
\hline \multicolumn{3}{|l|}{ Puntuación CBI } \\
\hline$M$ & 38.9 & \\
\hline$D T$ & 12.1 & \\
\hline
\end{tabular}

\section{Persona cuidada}

\begin{tabular}{lcc}
\hline Edad (años) & & \\
$M$ & 81.9 & \\
$D T$ & 6.1 & 69.2 \\
Sexo & 9 & 30.8 \\
Mujer & 4 & 23.1 \\
Hombre & & 15.4 \\
Grado de severidad de la demencia & 3 & 61.5 \\
Leve & 2 & 8 \\
Moderado & 8 & \\
Moderadamente grave o grave & 8 & \\
\end{tabular}

Nota. CES-D: Center for Epidemiologic Studies Depression Scale; SPSI-R: Social Problem-Solving Inventory-Revised; SAS-M: Social Adjustment Scale-Modified; CBI: Caregiver Burden Interview. 
estructural, concurrente, predictiva, convergente y discriminativa (D’Zurilla et al., 1997). En la adaptación al castellano la consistencia interna se sitúa entre 0.68 y 0.90 (Maydeu-Olivares et al., 2000).

Escala de Ajuste Social (Social Adjustment ScaleModified; SAS-M; Cooper, Osborn, Gath y Feggetter, 1982). Se trata de una escala autoadministrada que valora distintos aspectos del ajuste social del sujeto a lo largo de las últimas dos semanas. Consta de 45 ítems con una escala tipo Likert que va desde 1 (casi nunca) a 5 (todo el tiempo). Proporciona una puntuación global y siete áreas de la vida social del individuo: trabajo fuera de casa, tareas domésticas, actividades de ocio y tiempo libre, familia extensa, pareja, hijos y unidad familiar. En esta escala, el rango de puntuaciones está comprendido de 0 a 5 , donde unas mayores puntuaciones representan un mayor grado de deterioro social. Cooper et al. (1982) avalan un nivel satisfactorio de acuerdo entre la autoevaluación del sujeto y la puntuación del psiquiatra (índice kappa entre 0.52 y 0.80 ), así como una buena relación entre el SAS-M y la entrevista SAS (0.80). Para maximizar las equivalencias transculturales, la versión española de esta escala ha sido construida por nuestro equipo de investigación de acuerdo con las directrices de Guillermin, Bombardier y Beaton (1993), incluyendo el método de traducción retro-traducción (Brislin, Lonner y Thorndike, 1973), como sigue: fue traducida al español por dos investigadores (ambos nativos españoles e hispanoparlantes familiarizados con el inglés), y traducida al inglés por un traductor independiente, cuya lengua materna es el inglés y que previamente no había tenido contacto con el SAS-M. Esta traducción al inglés fue usada por un panel de expertos bilingüe para identificar y resolver los fallos en la versión española. La nueva versión fue traducida de nuevo al inglés y evaluada por el panel de expertos. Este proceso se repitió hasta que el panel de expertos consideró que la versión inglesa y la española eran equivalentes.

Entrevista de sobrecarga del cuidador (Caregiver Burden Interview; CBI; Zarit, Reever y BachPeterson, 1980; versión española de Martín et al., 1996). Consta de 22 ítems y evalúa la carga subjetiva asociada al cuidado. Las puntuaciones se valoran mediante una escala tipo Likert que va desde 0 (nunca) hasta 4 (casi siempre), con lo que el rango de la puntuación total que se puede alcanzar en el cuestionario es de 0 a 88. Uno de los ítems aporta información sobre el nivel global de sobrecarga. La adaptación de esta escala al castellano posee adecuadas propiedades psicométricas: una buena fiabilidad test-retest $(r=.86)$ y una satisfactoria consistencia interna $(\alpha=.91)$ (Martín et al., 1996).

\section{Tratamiento}

En esta intervención se parte de la premisa formulada por Nezu, Nezu y Perri (1989) de que los déficits en habilidades de solución de problemas suponen un factor de vulnerabilidad para la depresión. La depresión es más probable que ocurra cuando tienen lugar eventos vitales negativos y problemas cotidianos unidos a déficits en la capacidad de resolución de los mismos. La no resolución de estos problemas supone el mantenimiento de las consecuencias negativas que de ellos derivan y una fuente de estrés sostenido. Por tanto, el entrenamiento en solución de problemas conduce a una disminución de la sintomatología depresiva.

Puesto que la evidencia indica que los cuidadores informales experimentan numerosos eventos vitales negativos y problemas cotidianos en sus vidas (relacionados o no con las labores de cuidado), y además los sujetos seleccionados para este estudio presentaban elevada sintomatología depresiva, la terapia de solución de problemas parece ser adecuada para reducir su sintomatología depresiva y prevenir futuros episodios depresivos. Estudios anteriores han sugerido la eficacia de esta técnica en poblaciones de cuidadores y su asociación con menores niveles de sintomatología depresiva (Bourgeois, Schulz, Burgio y Beach, 2002; Lovett y Gallagher, 1988; Teri et al., 1997), así como su eficacia en el tratamiento de la depresión mayor (Vázquez, 2002).

Partiendo de este planteamiento y en este contexto, se desarrolló un programa de intervención breve basada en la terapia de solución de problemas con entrenamiento en los cinco componentes de la solución de problemas (orientación hacia el problema, definición del problema, generación de soluciones alternativas, toma de decisión y aplicación y verifi- 
cación de la solución escogida) de acuerdo a un material previamente estructurado (ver Tabla 2). Este programa de intervención fue aplicado en cinco intervención con el objeto de garantizar el cumplimiento de los criterios de inclusión y exclusión y establecer una línea base. Posteriormente, fueron

Tabla 2. Contenidos del programa de intervención de prevención de la depresión para cuidadores

\begin{tabular}{|c|c|c|}
\hline Sesión & Contenidos & Actividades \\
\hline \multirow[t]{6}{*}{ Sesión 1} & $\begin{array}{l}\text { Bienvenida. Presentaciones } \\
\text { Propósito del programa }\end{array}$ & \\
\hline & Explicación sobre la depresión & Discutir qué se entiende por depresión \\
\hline & & Analizar las causas de la depresión como resultado de un aprendizaje \\
\hline & Cómo puede ayudar el programa & $\begin{array}{l}\text { Presentar la técnica de solución de problemas y cómo influye en la depresión } \\
\text { Identificar los objetivos y componentes de la técnica }\end{array}$ \\
\hline & $\begin{array}{l}\text { Componentes de la orientación positiva hacia } \\
\text { el problema }\end{array}$ & Identificar la propia forma de ver los problemas, la atribución y el locus de control \\
\hline & Tarea para casa & Elaborar una lista de problemas actuales \\
\hline \multirow[t]{7}{*}{ Sesión 2} & Identificar el problema & Identificar algunos problemas actuales y elegir uno para trabajar en las sesiones \\
\hline & Definir el problema & Clarificar la naturaleza del problema \\
\hline & & Recopilar información sobre el problema \\
\hline & & Corregir distorsiones y errores cognitivos \\
\hline & Formulación del problema & Entrenar a los participantes en una formulación concreta del problema \\
\hline & & Descomponer el problema, si es necesario \\
\hline & Tarea para casa & Utilizar las técnicas para contrarrestar las distorsiones cognitivas \\
\hline \multirow[t]{3}{*}{ Sesión 3} & Establecer metas & $\begin{array}{l}\text { Pautas para establecer metas realistas } \\
\text { Reenfocar el problema }\end{array}$ \\
\hline & Generación de soluciones alternativas & $\begin{array}{l}\text { Conocer los principios para una generación de soluciones alternativas productiva } \\
\text { (cantidad, variedad, aplazamiento del juicio) }\end{array}$ \\
\hline & & Realizar una lluvia de ideas para generar soluciones alternativas \\
\hline \multirow[t]{5}{*}{ Sesión 4} & Toma de decisión & Realizar un análisis profundo de los costes y beneficios de cada solución alternativa \\
\hline & & Pautas para reconocer cuál es la solución más adecuada \\
\hline & & Tomar la decisión sobre la solución más adecuada \\
\hline & & Planificación de la aplicación de la solución escogida \\
\hline & Tarea para casa & Puesta en práctica de la solución elegida \\
\hline & & Reforzamiento tras la aplicaci \\
\hline
\end{tabular}

Sesión 5 Verificación de la solución escogida

Observar el resultado alcanzado con la solución escogida

Comparar el resultado actual y el que se esperaba

Analizar las dificultades encontradas

Valoración del programa

Despedida y cierre

sesiones de 1 hora y 30 minutos de duración aproximadamente, a razón de una sesión por semana. El formato utilizado para ello fue un formato grupal, con cuatro o cinco sujetos en cada grupo.

\section{Procedimiento}

En primer lugar se procedió a contactar con dos asociaciones federadas de familiares de enfermos de Alzheimer y otras demencias de Galicia, quienes nos facilitaron el acercamiento a los cuidadores pertenecientes a dichas asociaciones.

Todos los sujetos que formaron parte en el estudio fueron evaluados con anterioridad al inicio de la evaluados tras la terminación del programa de intervención y a los tres meses de su finalización para evaluar los efectos de la intervención.

Todas las evaluaciones fueron realizadas por evaluadores independientes. El proceso de evaluación de los cuidadores consistió en una entrevista clínica y la aplicación de la batería de cuestionarios anteriormente señalados. Toda la información se recogió con la garantía de confidencialidad de la información proporcionada.

La aplicación del programa fue llevada a cabo por tres licenciadas en psicología previamente entrenadas en la técnica de solución de problemas (dos de ellas con experiencia previa en esta modalidad de intervención). El análisis de los resultados obtenidos 
por los cuidadores en sintomatología depresiva en relación a la terapeuta que guió cada grupo mostró que no existen diferencias estadísticamente significativas en función de la terapeuta que aplicó el programa de intervención.

\section{Análisis estadístico}

Para el análisis de los datos se empleó el paquete estadístico SPSS para Windows (versión 15.0). Se calcularon las distribuciones de frecuencia y los estadísticos descriptivos para la muestra en conjunto. Para comparar dos medidas repetidas cuando ambas variables eran cualitativas se utilizó el test de McNemar. Cuando se compararon más de dos variables cuantitativas repetidas se utilizó un ANOVA de dos vías (sujeto y medida), en vez de un ANOVA de medidas repetidas, siguiendo las recomendaciones de Martínez-González, Sánchez-Villegas y Faulin (2006). Se reordenó la base de datos (sujeto, medida, CES-D) y se programó el modelo para que no exista interacción, pidiéndole al SPSS un modelo personalizado sin interacción. Para identificar dónde están las diferencias de medias entre las parejas de grupos se utilizó la prueba post hoc de Scheffé con un nivel de significación de .05.

\section{Resultados}

\section{Características de la muestra}

Las características sociodemográficas y clínicas más relevantes de los cuidadores que participaron en el estudio y de sus familiares con demencia pueden observarse en la Tabla 1. La edad media de los cuidadores fue de 59.9 años $(D T=11.9)$. El rango de edad comprendía desde los 43 a los 82 años. En cuanto al estado civil, el $76.9 \%$ está casado/a.

Por lo que respecta a la posición social, el $69.2 \%$ declararon pertenecer a una clase social media. Los ingresos mensuales por unidad familiar (ingresos recibidos cada mes por el conjunto de los miembros de la familia que viven juntos) se situó en la mayor parte de los casos (46.2\%) entre los $1000 \mathrm{y}$ $\operatorname{los} 2000 €$.
En la faceta de formación y situación laboral, se puede observar que un $61.5 \%$ de los cuidadores poseían estudios secundarios, seguidos de un $23.1 \%$ con estudios primarios. Con respecto a la actividad profesional, un $69.2 \%$ no estaban empleados, destacando en este grupo quienes se dedicaban a las tareas domésticas (46.1\%), seguidas por los cuidadores jubilados $(23.1 \%)$. Sólo una tercera parte de los cuidadores de la muestra se encontraban activos profesionalmente.

La relación que unía al cuidador con la persona que cuida fue mayoritariamente de tipo familiar y de primer grado, con un mayor porcentaje de hijos/as que proporcionaron cuidados a sus padres $(61.5 \%)$, seguido por los esposos/as al cuidado de sus parejas (30.8\%). Lo más habitual es que fuese la hija $(46.1 \%)$ o la esposa $(23.1 \%)$, frente a los hijos $(15.4 \%)$ o los esposos $(7.7 \%)$.

El tiempo dedicado al cuidado del ser querido fue intensivo y continuado. En esta muestra se recogió una media de 13.8 horas diarias dedicadas a los cuidados del familiar durante una media de 5.6 años. Pero además, a este acompañamiento en el tiempo se une el del espacio. La gran mayoría (el 92.3\%) informaron vivir con la persona cuidada.

La puntuación media de la sintomatología depresiva evaluada a través de la CES-D fue de 26.3 (DT =9.1). Por su parte, la puntuación media de las habilidades de solución de problemas fue de 85 ( $D T=$ 12.3). La puntuación media en ajuste social se situó en un $2.4(D T=0.4)$ y en sobrecarga en un $38.9(D T$ $=12.1)$.

Los familiares enfermos de demencia que son cuidados por los cuidadores de esta muestra tenían una edad media de 81.9 años $(D T=6.1)$ y fueron mayoritariamente mujeres $(69.2 \%)$. El grado de severidad, deterioro y dependencia producido por la demencia de la que son víctimas se considera como moderadamente grave o grave en el $61.5 \%$ de los casos.

\section{Resultados de la intervención}

En la Tabla 3 pueden observarse las puntuaciones obtenidas en las variables analizadas en los tres momentos de medida (pre-intervención, post-intervención y seguimiento). 
Sintomatología depresiva. En aquellos sujetos que completaron el programa de intervención, los cambios experimentados en las puntuaciones media en sintomatología depresiva en los distintos momentos de medida revelaron diferencias estadísticamente significativas, $F(2,24)=13.34, p<.001$. Tras participar en el programa de intervención, así como en el seguimiento, los cuidadores informales mostraron una menor puntuación media en sintomatología depresiva. Los análisis post hoc (prueba de Scheffé; $\alpha=.05$ ) indicaron que las diferencias estadísticamente significativas se encontraron entre las medias de la medida de pre-intervención y de postintervención $(p=.01)$ y de pre-intervención y seguimiento $(p<.001)$. No así entre la de post-intervención y la de seguimiento $(p=.19)$.

Pero además de un análisis del cambio de los niveles de sintomatología depresiva, se han estudiado los resultados en cuanto al riesgo de sufrir una depresión clínica (puntuaciones CES-D $\geq 16$ ). En la evaluación post-intervención seis sujetos experimentaron esta reducción del riesgo, lo que supone un $46.2 \%$ del total de sujetos. Esto alcanzó niveles de significación estadística $(p=.03)$. Tres meses más tarde, estos seis sujetos siguieron manteniendo sus resultados y se añadieron tres sujetos más, lo que supone un $23 \%$ más del total de sujetos de la muestra. Aunque las diferencias entre el postratamiento y los 3 meses de seguimiento no fueron significativos $(p=.25)$; al comparar la medida de pre-intervención con los tres meses de seguimiento también se hallaron diferencias estadísticamente significativas $(p=$ .004). Así, tras la intervención y los tres meses de seguimiento, un total de 9 sujetos $(69.2 \%$ de la muestra) obtuvieron puntuaciones inferiores a 16 en la CES-D, con una consecuente reducción del riesgo de sufrir una depresión clínica.

Habilidades de solución de problemas. Se encontraron diferencias estadísticamente significativas entre las puntuaciones medias obtenidas en el SPSI$\mathrm{R}$ en los distintos momentos de medida, $F(2,24)=$ $16.66, p<.001$. Las puntuaciones medias en el SPSI$\mathrm{R}$ que obtuvieron los sujetos que participaron en el estudio experimentaron un aumento paulatino a lo

Tabla 3. Comparación de las puntuaciones en las variables de resultado antes y después de la intervención y en la fase de seguimiento $(N=13)$

\begin{tabular}{|c|c|c|c|c|c|}
\hline Medida & Línea Base & Post-intervención & $\begin{array}{l}\text { Seguimiento } \\
\text { (3 meses) }\end{array}$ & & \\
\hline & $M(D T)$ & $M(D T)$ & $M(D T)$ & $F$ & $p$ \\
\hline Depresión (CES-D) & $26.3(9.1)$ & $18.2(7.3)$ & $13.4(4.7)$ & 13.34 & $<0.001$ \\
\hline $\begin{array}{l}\text { Habilidades de solución problemas } \\
\text { (SPSI-R) }\end{array}$ & $85(12.2)$ & $92.8(9.7)$ & $95.2(9)$ & 16.66 & $<0.001$ \\
\hline $\begin{array}{l}\text { Orientación positiva } \\
\text { Orientación negativa } \\
\text { Solución racional } \\
\text { Estilo impulsivo } \\
\text { Estilo evitativo } \\
\text { Definición problema } \\
\text { Soluciones alternativas } \\
\text { Toma de decisión } \\
\text { Verificación }\end{array}$ & $\begin{array}{r}81.8(14.7) \\
118.9(22.3) \\
88.5(14.6) \\
103.6(20.0) \\
106.9(15.7) \\
86.9(16.7) \\
86.5(13.5) \\
91.1(15.0) \\
92.8(15.4)\end{array}$ & $\begin{array}{r}85.1(15.2) \\
111.1(15.9) \\
92.5(13.4) \\
95.6(14.9) \\
100.5(8.1) \\
86.6(14) \\
91.8(13.3) \\
98.6(16.8) \\
96.6(16.1)\end{array}$ & $\begin{array}{r}85.9(14.7) \\
106.5(12.4) \\
93.2(14.1) \\
89.7(9.9) \\
100.1(9) \\
87.1(13.3) \\
89.7(24.5) \\
102.4(13.1) \\
99.5(15.9)\end{array}$ & $\begin{array}{r}1.77 \\
6.05 \\
1.97 \\
10.85 \\
4.78 \\
0.01 \\
1.88 \\
4.59 \\
3.89\end{array}$ & $\begin{aligned} & 0.19 \\
& 0.01 \\
& 0.16 \\
&< 0.001 \\
& 0.02 \\
& 0.99 \\
& 0.17 \\
& 0.02 \\
& 0.04\end{aligned}$ \\
\hline $\begin{array}{l}\text { Ajuste social } \\
\text { (SAS-M) }\end{array}$ & $2.4(0.4)$ & $2.2(0.2)$ & $2(0.3)$ & 15.27 & $<0.001$ \\
\hline $\begin{array}{l}\text { Trabajo } \\
\text { Tareas domésticas } \\
\text { Act. sociales y ocio } \\
\text { Familia extensa } \\
\text { Pareja } \\
\text { Hijos } \\
\text { Unidad familiar }\end{array}$ & $\begin{array}{l}2.2(0.7) \\
1.9(0.7) \\
2.8(0.6) \\
2.6(0.5) \\
2.7(0.5) \\
1.6(0.6) \\
2.4(0.9)\end{array}$ & $\begin{array}{l}1.8(0.6) \\
1.8(0.4) \\
2.8(0.5) \\
2.3(0.5) \\
2.5(0.5) \\
1.3(0.2) \\
2.2(0.7)\end{array}$ & $\begin{array}{l}1.5(0.4) \\
1.6(0.3) \\
2.5(0.4) \\
2.4(0.5) \\
2.5(0.6) \\
1.2(0.2) \\
1.6(0.5)\end{array}$ & $\begin{array}{l}5.93 \\
2.75 \\
2.26 \\
1.59 \\
0.87 \\
4.59 \\
5.35\end{array}$ & $\begin{array}{l}0.03 \\
0.08 \\
0.13 \\
0.23 \\
0.44 \\
0.03 \\
0.02\end{array}$ \\
\hline Sobrecarga (CBI) & $38.9(12.1)$ & $34.1(12.6)$ & $30.4(9)$ & 4.18 & 0.03 \\
\hline
\end{tabular}

Nota. CES-D: Center for Epidemiologic Studies Depression Scale; SPSI-R: Social Problem-Solving Inventory-Revised; SAS-M: Social Adjustment Scale- Modified; CBI: Caregiver Burden Interview; Orientación positiva: Orientación positiva hacia el problema; Orientación negativa: Orientación negativa hacia el problema; Solución racional: Solución de problemas racional; Soluciones alternativas: Generación de soluciones alternativas; Verificación: Implementación y verificación de la solución escogida; Act. Sociales y ocio: Actividades sociales y de ocio. 
largo de los distintos momentos de medida. La prueba post hoc de Scheffé ( $\alpha=.05)$ reveló que los cambios estadísticamente significativos ocurrieron entre la medida pre-intervención y la post-intervención ( $p$ $=.001) \mathrm{y}$ entre la medida pre-intervención y la de seguimiento $(p<.001)$. Aunque no se alcanzaron diferencias estadísticamente significativas entre la medida de post-intervención y la de seguimiento ( $p=$ .42 ), se observa un aumento de la puntuación media.

Cabe destacar aquí que la puntuación media en la línea base de los cuidadores se encontraba ligeramente por debajo de la puntuación media para su grupo normativo (86 - 114); aspecto que mejoró tras la intervención, cuando la puntuación media se situó dentro el rango establecido para las puntuaciones medias normales del grupo normativo.

Un análisis más profundo y pormenorizado de las nueve dimensiones que proporciona el SPSI-R nos ha permitido saber exactamente en qué componentes de las habilidades de solución de problemas se encuentran estas mejorías, así como en qué momento del proceso tuvieron lugar. Así, se hallaron diferencias estadísticamente significativas entre los distintos momentos de medida en la orientación negativa hacia el problema, $F(2,24)=6.05, p<.01$, en el estilo impulsivo, $F(2,24)=10.85, p<.001$, en el estilo evitativo, $\mathrm{F}(2,24)=4.78, p<.05$, en la toma de decisiones, $F(2,24)=4.59, p<.05$ y en la implementación y verificación de la solución elegida, $F(2$, $24)=3.89, p<.05 . \mathrm{Al}$ realizar las pruebas post hoc (Scheffé; $\alpha=.05$ ) se encontró que hubo diferencias estadísticamente significativas entre la medida de pre-intervención y la de post-intervención en los estilos impulsivo y evitativo (ambos con $p<.05$ ). Estas diferencias estadísticamente significativas se mantuvieron entre la medida pre-intervención y la de seguimiento (estilo impulsivo, $p<.001$; estilo evitativo, $p$ $<.05$ ), pero no ocurrieron entre la medida de postintervención y la de seguimiento (estilo impulsivo, $p$ $=.16$; estilo evitativo, $p=.9$ ). Las diferencias estadísticamente significativas entre la pre-intervención y el seguimiento también se pudieron observar en la orientación negativa hacia el problema $(p<.01)$, la toma de decisiones $(p<.05)$ y la implementación y verificación de la solución $(p<.05)$.

Por último, al analizar la evolución de la sintomatología depresiva y de las habilidades de solución de problemas a través del tiempo, se observa una tendencia de disminución de las puntuaciones en sintomatología depresiva en consonancia con un aumento de las puntuaciones en habilidades de solución de problemas.

Otros efectos de la intervención. Con respecto al ajuste social, se encontraron diferencias estadísticamente significativas entre las puntuaciones medias globales en ajuste social en los distintos momentos de medida, $F(2,24)=15.27, p<.001$, con puntuaciones medias progresivamente menores. Más concretamente, según los análisis post hoc (prueba de Scheffé, $\alpha=.05$ ), las diferencias se hallaron entre la medida de pre-intervención y la de post-intervención $(p<.01)$, y entre la de pre-intervención y la de seguimiento $(p<.001)$. En cambio, las diferencias de medias existentes entre la medida post-intervención y la de seguimiento no alcanzaron a ser estadísticamente significativas $(p=.17)$.

Siguiendo un análisis más detallado, por áreas, se encontraron diferencias estadísticamente significativas en las siguientes áreas sociales: trabajo fuera de casa, $F(2,8)=5.93, p<.05$, relaciones con los hijos, $F(2,14)=4.59, p<.05$ y unidad familiar, $F$ $(2,16)=5.35, p<.05$. En todos los casos, estas diferencias se produjeron entre la medida pre-intervención y la de seguimiento $(p<.05)$.

Con respecto a la sobrecarga experimentada por los cuidadores, las diferencias de medias en los tres momentos de medida también alcanzaron diferencias estadísticamente significativas, $F(2,21)=4.18$, $p=.03$. Nuevamente, las puntuaciones medias en los tres momentos de medida mostraron una disminución paulatina. Pero en este caso, la prueba post hoc de Scheffé ( $\alpha=.05$ ) sólo encontró estas diferencias estadísticamente significativas entre la medida pre-intervención y la de seguimiento $(p=.02)$. Entre la pre-intervención y la post-intervención y entre ésta y la de seguimiento no se hallaron diferencias estadísticamente significativas $(p=.20$ y $p=.48$, respectivamente).

\section{Discusión}

Este estudio piloto tenía como objetivo principal evaluar la viabilidad de un programa de intervención 
breve para prevenir la depresión en los cuidadores informales de personas con demencia que presentaban sintomatología depresiva sin llegar a cumplir los criterios diagnósticos para el trastorno de depresión mayor (prevención indicada).

Se encontró que los cuidadores que participaron en este estudio disminuyeron significativamente el nivel de sintomatología depresiva tras la aplicación de la intervención y mantuvieron dicha reducción a los tres meses de seguimiento. De igual forma, se alcanzó una reducción significativa del riesgo de desarrollar una depresión clínica tras la intervención, cuyos efectos se prolongaron hasta tres meses después.

Los resultados alcanzados en reducción de la sintomatología depresiva son congruentes con los hallados en diversos estudios en los que la aplicación de diversos programas de intervención para reducir la sintomatología depresiva alcanzaron resultados estadísticamente significativos (ej., Coon et al., 2003; Eisdofer et al., 2003; MárquezGonzález, Losada, Izal, Pérez-Rojo y Montorio, 2007). Pero por otra parte, estos resultados fueron más positivos que los hallados en los estudios de Burns, Nichols, Martindale-Adams, Graney y Lummus (2003), Farran et al. (2004), Gendron, Poitras, Dastoor y Pérodeau (1996), Haley, Brown y Levine (1987), Hébert, Leclerc, Bravo, Girouard y Lefrançois (1994), Losada-Baltar, Izal-Fernández de Trocóniz, Montorrio-Cerrato, Márquez-González y Pérez-Rojo (2004) y Zanetti, Metitieri, Bianchetti y Trabucchi (1988), en los que no se consiguió reducir la sintomatología depresiva de los cuidadores.

Es más, este estudio superó con sus resultados lo esperado por algunos autores con respecto a la reducción de la sintomatología depresiva. Así, López y Crespo (2007), en su revisión de intervenciones con cuidadores, manifiestan que la depresión parece el resultado más difícil de modificar, siendo la única medida que en ciertos estudios incrementaba sus niveles tras la intervención. Por su parte, Gottlieb, Thompson y Bourgeois (2003) argumentan que es de esperar que los cuidadores manifiesten síntomas depresivos sin que se produzcan reducciones significativas a causa de la intervención, dado que ésta no cambia la situación de la enfermedad de su familiar; y que en estas circunstancias de males- tar crónico se consideraría un logro el conseguir que el malestar no aumentase.

Por otra parte, se pudo constatar como las habilidades de solución de problemas experimentaron una mejoría significativa tras la aplicación del programa de intervención, que pasaron de un nivel ligeramente inferior al normal esperado para su grupo normativo de edad al rango normal esperado (86 - 114). Los aspectos de la solución de problemas más beneficiados por el programa de intervención fueron la orientación negativa hacia el problema y los estilos impulsivo y evitativo como modo de afrontamiento, que se vieron disminuidos, y los procesos de toma de decisiones y de implementación y verificación de la solución, que se vieron aumentados. Esto supone que tras la intervención, los cuidadores participantes en este estudio empezaron a apreciar los problemas de una forma menos negativa, a enfrentarse a sus problemas sin precipitarse ni evitarlos, sopesar mejor los pros y los contras de las posibles soluciones para escoger la más adecuada y evaluar los resultados obtenidos con esa solución.

Estos resultados, sin embargo, no se pueden comparar con estudios anteriores, pues aunque algunos estudios también han empleado la técnica de solución de problemas en sus programas de intervención con cuidadores de personas con demencia (Bourgeois et al., 2002; Gendron et al., 1996; Losada-Baltar et al., 2004; Lovett y Gallagher, 1988; Teri et al., 1997), ninguno de ellos evaluó las habilidades de solución de problemas como variable de resultado.

Cabe mencionar que los resultados hallados en este estudio permiten recoger una tendencia de reducción de la sintomatología depresiva que se corresponde con un aumento en las habilidades de solución de problemas. Aunque estos datos deben ser tomados con cautela debido al pequeño tamaño de la muestra, los resultados conseguidos en la reducción de la sintomatología depresiva están en consonancia con la aplicación del programa de intervención y el aprendizaje y mejoría de las habilidades de solución de problemas planteadas en él. Probablemente, con un tamaño de muestra mayor la capacidad de solución de problemas se comportaría como una variable moduladora de la sintomatología depresiva. 
Una posible causa de la mejora de la sintomatología depresiva en el presente estudio con respecto a otros estudios podría estribar en la adecuación del programa de intervención a las necesidades específicas de los cuidadores como individuos, además de las relacionadas con su condición de cuidadores. Así, por ejemplo, mientras en anteriores estudios la técnica de solución de problemas había estado focalizada en enseñar a los cuidadores a manejar los problemas estrictamente relacionados con la enfermedad del familiar o con los problemas de las tareas de cuidado, en el presente estudio se entrenó a los cuidadores para resolver de forma racional y sistemática todo tipo de problemas, alcanzando así soluciones de más calidad (Muñoz Adanes, 2005), independientemente de si los problemas estaban relacionados o no con la labor de cuidado. De esta forma, la intervención no se centra en los problemas relacionados con la demencia del familiar, los cuales pueden producir un aumento del malestar del cuidador por tratarse de problemas crónicos e irresolubles (Zarit, Zarit y Reever, 1982).

Por otra parte, se hallaron también mejorías significativas en el ajuste social y la sobrecarga experimentada por los cuidadores tras la intervención y el seguimiento. Tal como se propone desde el campo de prevención de la depresión (Muñoz, Le, Clarke y Jacox, 2002), las mejorías se evaluaron y se produjeron, no sólo en la sintomatología depresiva, sino también en otras áreas de la vida del individuo, garantizando así un efecto de generalización de los resultados. Con respecto al ajuste social, se produjo una disminución significativa del deterioro social de los cuidadores tras la intervención que se mantuvo hasta los tres meses de seguimiento. Las áreas de la vida de los cuidadores que mostraron una reducción significativa en su deterioro fueron el trabajo fuera de casa, las relaciones con los hijos y la unidad familiar. En cuanto a la sobrecarga, los cuidadores experimentaron una menor sobrecarga a los tres meses de seguimiento.

Ninguno de los estudios anteriores llevados a cabo con cuidadores informales de personas con demencia seleccionó la muestra en base a los factores de riesgo que presentan, pese a que los grupos de alto riesgo con factores de riesgo múltiples son los más apropiados para llevar a cabo una intervención de tipo preventivo (Cuijpers, 2003). A diferencia de esto, el presente estudio se centra en la prevención de la depresión en una población con factores de riesgo múltiple. Los factores de riesgo aquí seleccionados fueron el hecho de ser cuidador informal que, además, presentaba niveles elevados de síntomas depresivos, cuestiones ambas relacionadas con un riesgo elevado de desarrollar una depresión clínica (Covinsky et al., 2003; Moral Serrano et al., 2003; Cuijpers y Smit, 2004; OMS, 2004).

En vez de esto, los estudios anteriores mezclaron en la misma muestra cuidadores sin sintomatología depresiva, con sintomatología depresiva y cuidadores ya clínicamente deprimidos (Teri et al., 1997), con distintos niveles de sintomatología depresiva (Eisdofer et al., 2003; Gendron et al., 1996), o cuidadores con alto riesgo en los que se identificaron manifestaciones prodrómicas de la depresión con otros en los que no se daban estas circunstancias (Burns et al., 2003; Gallagher-Thompson et al., 2003). Esto supone un descuido de las necesidades clínicas que demanda cada perfil de riesgo, pues es difícil cubrir con el mismo programa de intervención las necesidades presentes en sujetos sin sintomatología depresiva, sujetos con sintomatología depresiva pero que no cumplen los criterios para un trastorno depresivo mayor y sujetos que ya padecen un trastorno depresivo mayor. Además, a nivel metodológico, al estar evaluando de forma conjunta a sujetos con sintomatología depresiva y sujetos sin esta sintomatología, se puede crear un efecto suelo que impida registrar mejorías (López y Crespo, 2007).

Como resultado del análisis del estudio piloto, se sugiere la realización de ciertas modificaciones que puedan repercutir en la mejora de la aceptabilidad y puesta en práctica del programa de intervención. Estas modificaciones no se refieren a contenidos esenciales del programa de intervención, que mantendría su formato original, sino fundamentalmente a la reducción de los materiales e instrumentos de evaluación con el objeto de favorecer su aceptabilidad. Así, se sugiere la reducción del número de tareas para casa y de los contenidos del material escrito entregado a los cuidadores como resúmenes de las sesiones, puesto que la mayoría de los sujetos manifestaron que no siempre disponían de suficiente tiempo para su realización y lectura debido a la 
sobrecarga de tareas que experimentan. Se recomienda la realización de algunas tareas durante el tiempo de la sesión y la asignación de tareas para casa más aplicadas a la vida cotidiana de los cuidadores. También se sugiere la reducción del número de instrumentos de evaluación para evitar la fatiga de los cuidadores, y la ampliación de las medidas de seguimiento a seis y doce meses para valorar el alcance de los resultados de la intervención.

Este estudio viene a actualizar los conocimientos sobre las intervenciones en cuidadores informales en situación de riesgo de depresión, dando luz a parcelas antes no estudiadas al iniciar la investigación sobre la prevención indicada de la depresión en cuidadores, y sentando las bases para posibles mejoras en las intervenciones futuras. Los resultados obtenidos respaldan las hipótesis planteadas y sugieren la viabilidad del programa de intervención propuesto. Se trata de un programa fundamentado teóricamente pero que al mismo tiempo tiene en cuenta su aplicabilidad y realiza una selección de la muestra para facilitar una adecuación a las necesidades individua- les de los cuidadores y no sólo a sus necesidades como colectivo. Quizás la ausencia de estos elementos en los anteriores estudios hayan derivado en los efectos relativamente pequeños que se pueden observar en las intervenciones realizadas hasta la actualidad con cuidadores informales de personas con demencia.

Sin embargo, hay que tener en cuenta que el estudio que aquí se presenta es un estudio piloto, una primera toma de contacto con el objeto de estudio con una muestra reducida para determinar su viabilidad para estudios posteriores. Por ello es conveniente ser prudente con los resultados obtenidos, pues dado el pequeño tamaño de la muestra, podrían no ser generalizables y concluyentes. Además, puesto que este estudio carece de grupo control, se desconocen los cambios que hubieran tenido lugar sin la intervención. Son necesarias futuras investigaciones con una muestra más amplia y un diseño aleatorio y controlado que pudieran corroborar los resultados aquí hallados y evaluar la eficacia, efectividad y coste-eficacia de este tipo de intervención.

\section{Extended Summary}

Individuals who care for severely handicapped dependants are at high risk of depression. There have been reports that $40-70 \%$ of informal caregivers have significant levels of clinically relevant symptoms, and that about $50 \%$ may satisfy diagnostic criteria for major depressive disorder (MDD). In response to this situation, a number of psychological intervention programmes have been devised specifically for informal caregivers, especially those caring for people with dementia. So far, however, few such programmes have actually been evaluated in terms of their influence on measures of depressive symptoms, and none of those evaluated has been aimed specifically at preventing depression in dementia patient caregivers. Furthermore, most programmes for dementia patient caregivers have not been based on any definite theoretical model. In this study we evaluated a short problem-solving based intervention programme designed to prevent the progression of depression in dementia patient caregivers with sub-MDD symptoms of depression.

The caregivers taking part in the study were recruited from among members of associations belonging to FAGAL, the Federation of Galician Associations of Relatives of Persons with Alzheimer's Disease or Other Dementias. Potential participants were contacted personally, informed of the nature of the study, invited to participate and assured of confidentiality and anonymity, and all their queries were answered to their satisfaction. Participation was totally voluntary, without any economic or other extraneous incentive. Each potential participant was required $a$ ) to be an informal carer of a dependent relative with medically diagnosed dementia (not necessarily Alzheimer's disease), and $b$ ) to undergo a preliminary psychological evaluation protocol involving self-report questionnaires and an interview with a psychologist. Candidates were admitted to the study if they $c$ ) had a score of 16 or more (indicative of risk of depression) on the Centre for Epidemiologic Studies Depression Scale $(\mathrm{CES}-\mathrm{D}), d)$ agreed to undergo the necessary evaluations, and $e$ ) gave written informed consent to the application of the prevention programme. 
The final study group included 13 caregivers (10 women and 3 men) with ages ranging from 43 to 82 ( $M=59.9 ; S D=11.9$ years). The intervention programme consisted of training to strengthen their abilities in regard to the five aspects of problem-solving recognized by social problem-solving theory: attitude towards problems, problem definition, generation of alternative possible solutions, choice of a solution, and execution and verification of the solution chosen. The programme was administered by three trained psychologists in accordance with a predesigned structured protocol in five 90-minute group sessions, with four or five participants per group. Participants were evaluated by clinical interview and questionnaires at three stages: before the start of the programme, at its conclusion, and 3 months after its conclusion - and never by any of the three psychologists who administered the programme.

The instruments employed were a questionnaire recording sociodemographic data, the CES-D, the clinical version of the Structured Clinical Interview for DSM-IV Axis I Disorders (SCID-CV), the revised Social Problem-Solving Inventory (SPSIR), the modified Social Adjustment Scale (SAS-M), and the Caregiver Burden Inventory (CBI).

Some $77 \%$ of participants were women, $77 \%$ were married, $69 \%$ middle-class, and $69 \%$ unemployed; $62 \%$ had received secondary education, and $69 \%$ devoted most of their time to household chores and caring for their parents. Caring for the carerequired occupied an average 13.8 hours daily, and had been performed for an average 5.6 years. Twelve of the participants (92\%) lived with the person cared for.

An ANOVA of the three sets of evaluations showed statistically significant between-evaluation differences in depressive symptoms $(p<.001)$, social adjustment $(p<.001)$, caregiving burden $(p$ $<.05)$, and problem-solving skills $(p<.001)$. More specifically, post hoc comparison of pre- and immediately post-intervention data showed reductions in depressive symptoms $(p=.01)$ and social malfunction $(p<.01)$ and an increase in problemsolving skills $(p=.001)$; and comparison of preintervention and 3-month follow-up data showed reductions in depressive symptoms $(p<.001)$, social malfunction $(p<.001)$ and caregiving bur- den $(p=.001)$, and increased problem-solving skills $(p<.02)$. With regard to specific SPSI-R components, there were significant between-evaluation differences in negative problem orientation $(p$ $<.01)$, impulsivity/carelessness style $(p<.001)$, avoidance style $(p<.05)$, decision making $(p<$ $.05)$, and implementation and verification of solutions $(p<.05)$. With regard to specific SASM components, there were significant between-evaluation differences in the areas of work, parenting and extended family ( $p<.05$ in all cases).

The above results show that the caregivers who participated in this study had significantly lower levels of depressive symptoms and risk of depression after the intervention than before, and that these reductions were maintained for at least 3 months. Most evaluation studies of other psychological intervention programmes for caregivers (though not all) have not observed significant reductions in depressive symptoms. The better performance of the present programme may be attributable in part to its aiming to improve participants' general problemsolving skills rather than limiting attention specifically to caregiving issues. The caregivers' problemsolving skills were indeed significantly greater after the programme than before, especially in the areas of negative problem orientation, impulsivity/carelessness style, avoidance style, decision making, and implementation and verification of solutions. As far as we know, this is the first problem-solving based depression-prevention programme for dementia patient carers for which improvement in problemsolving skills has actually been evaluated. However, it should be borne in mind that, curiously enough, in no other study of a psychological intervention programme for dementia patient carers were participants selected on the basis of their risk of depression: the samples in these studies included, without distinction, caregivers with and without symptoms of depression or clinically diagnosed depressive disorder, or with depressive symptoms ranging from minor indications to prodromic manifestations. Thus, the encouraging results of the present programme may be partly due to its having been designed for caregivers with a specific level of risk, and applied to subjects belonging to the category for which it was designed. Programmes that afford the 
same treatment to caregivers with widely differing levels of depressive symptoms may be attempting the impossible.

Our results suggest the utility of the proposed intervention programme, with or without minor modifications implemented in light of our experience so far (e.g. a reduction in homework). The observed significant improvements in the participating caregivers' social functioning, and reduction of their caregiving burden, are especially encouraging, since they show that the beneficial effects of the programme extended beyond the specific issues of depression to other aspects of the participants' lives. It must nevertheless be borne in mind that this was a pilot study with a very small number of participants and no control group. Randomized controlled studies with larger samples will be necessary to confirm the present results.

\section{Referencias}

American Psychiatric Asocciation (1995). Manual diagnóstico y estadístico de los trastornos mentales (Cuarta Edición). Barcelona: Masson.

Bergman-Evans, B. (1994). A health profile of spousal Alzheimer's caregivers: Depression and physical health characteristics. Journal of Psychosocial Nursing and Mental Health Services, 32, 25-30.

Bourgeois, M.S., Schulz, R., Burgio, L.D. y Beach, S. (2002). Skills training for spouses of patients with Alzheimer's disease: Outcomes of an intervention study. Journal of Critical Geropsychology, 8, 53-73.

Brislin, R.W., Lonner, W.J. y Thorndike, E.M. (1973). Cross-cultural research methods. New York: Wiley.

Brodaty, H., Green, A. y Koschera, A. (2003). Metaanalysis of psychosocial interventions for caregivers of people with dementia. Journal of the American Geriatrics Society, 51, 657-664.

Burns, R., Nichols, L.O., Martindale-Adams, J., Graney, M.J. y Lummus, A. (2003). Primary care interventions for dementia caregivers: 2-year outcomes from the REACH study. Gerontologist, 43, 547-555.
Carrillo, J.M., Rojo, N. y Staats, A.W. (2004). Women and vulnerability to depression: Some personality and clinical factors. Spanish Journal of Psychology, 1, 29-39.

Chang, B.L. (1999). Cognitive-behavioral intervention for homebound caregivers of persons with dementia. Nursing Research, 48, 173-182.

Coon, D.W., Thompson, L., Steffen, A., Sorocco, K. y Gallagher-Thompson, D. (2003). Anger and depression management: Psychoeducational skill training interventions for women caregivers of a relative with dementia. Gerontologist, 43, 678689.

Cooper, P., Osborn, M., Gath, D. y Feggeter, G. (1982). Evaluation of a Modified Self-Report Measure of Social Adjustment. British Journal of Psychiatry, 141, 68-75.

Covinsky, K.E., Newcomer, R., Fox, P., Wood, J., Sands, L., Dane, K. et al. (2003). Patient and caregiver characteristics associated with depression in caregivers of patients with dementia. Journal of General Internal Medicine, 18, 10061014.

Cuijpers, P. (2003). Examining the effects of prevention programs on the incidence of new cases of mental disorders: The lack of statistical power. The American Journal of Psychiatry, 160, 13851391.

Cuijpers, P. y Smit, F. (2004). Subthreshold depression as a risk indicator for major depressive disorder: A systematic review of prospective studies. Acta Psychiatrica Scandinavica, 109, 325-331.

Dozois, D.J.A. y Dobson, K.S. (2004). The prevention of anxiety and depression: Introduction. En D.J.A. Dozois y K.S. Dobson (Eds.), The prevention of anxiety and depression: Theory, research and practice (pp. 1-6). Washington, DC: American Psychological Association.

D'Zurilla, T.J., Nezu, A.M. y Maydeu-Olivares, A. (1997). Manual for the social problem solving inventory-revised. New York: Multi-Health Systems.

Eisdofer, C., Czaja, S., Loewenstein, D.A., Rubert, M.P., Argüelles, S., Mitrani, V.B. et al. (2003). The effect of a family therapy and technologybased intervention on caregiver depression. Gerontologist, 43, 521-531. 
Farran, C.J., Gilley, D.W., McCann, J.J., Bienias, J.L., Lindeman, D.A. y Evans, D. A. (2004). Psychological interventions to reduce depressive symptoms of dementia caregivers: A randomized clinical trial comparing two approaches. Journal of Mental Health and Aging, 10, 337-350.

First, M.B, Spitzer, R.L., Gibbon, M. y Williams, J.B.W (1999). Entrevista clínica estructurada para los trastornos del Eje I del DSM-IV, Versión Clínica (SCID-VC). Barcelona: Masson.

Gallagher, D., Rose, J., Rivera, P., Lovett, S. y Thompson, L.V. (1989). Prevalence of depression in family caregivers. Gerontologist, 29, 449-456.

Gallagher-Thompson, D., Coon, D., Solano, N., Ambler, C., Rabinowitz, Y. y Thompson, L.W. (2003). Change in indices of distress among Latino and Anglo females caregivers of elderly relatives with dementia: Site-specific results from the REACH National Collaborative Study. Gerontologist, 43, 580-591.

Gallagher-Thompson, D., Lovett, S., Rose, J., McKibbin, C., Coon, D.W., Futterman, A. et al. (2000). Impact of psychoeducational interventions on distressed family caregivers. Journal of Clinical Geropsychiatry, 2, 91-110.

Garand, L., Dew, M.A., Eazor, L.R., DeKoski, S.T. y Reynolds, C.F. (2005). Caregiving burden and psychiatric morbidity in spouses of persons with mild cognitive impairment. International Journal of Geriatric Psychiatry, 20, 512-522.

Gendron, C., Poitras, L., Dastoor, D. P. y Pérodeau, G. (1996). Cognitive-behavioral group intervention for spousal caregivers: Findings and clinical considerations. Clinical Gerontologist, 17, 3-19.

Gilley, D.W., McCann, J.J., Bienias, J.L. y Evans, D.A. (2005). Caregiver psychological adjustment and institutionalization of persons with Alzheimer's disease. Journal of Aging and Health, 17, 172-189.

Gottlieb, B.H., Thompson, L.W. y Bourgeois, M. (2003). Monitoring and evaluating interventions. En D.W. Coon, D. Gallagher-Thompson y L.W. Thompson (Eds.), Innovative interventions to reduce dementia caregiver distress (pp. 28-49). New York: Springer.

Guillermin, F., Bombardier, C. y Beaton, D. (1993). Cross-cultural adaptation of health related quality of life measures: Literature review and proposed guidelines. Journal of Clinical Epidemiology, 43, 1417-1432.

Haley, W. E., Brown, S.L. y Levine, E.G. (1987). Experimental evaluation of the effectiveness of group intervention for dementia caregivers. Gerontologist, 27, 376-382.

Hébert, R., Leclerc, G., Bravo, G., Girouard, D. y Lefrançois, R. (1994). Efficacy of a support group programme for caregivers of demented patients in the community: A randomized controlled trial. Archives of Gerontology and Geriatrics, 18, 1-14.

López, J. y Crespo, M. (2007). Intervenciones con cuidadores de familiares mayores dependientes: una revisión. Psicothema, 19, 72-80.

Losada-Baltar, A., Izal-Fernández de Trocóniz, M., Montorrio-Cerrato, I., Márquez-González, M. y Pérez-Rojo, G. (2004). Eficacia diferencial de dos intervenciones psicoeducativas para cuidadores de familiares con demencia. Revista de Neurología, 38, 701-708.

Lovett, S. y Gallagher, D. (1988). Psychoeducational interventions for family caregivers: Preliminary efficacy data. Behavior Therapy, 19, 321330 .

Maidment, R., Regan, C., Katona, C. y Livingston, G. (2005). Anxiety and depression in family caregivers of people with Alzheimer disease: The LASER-AD study. The American Journal of Geriatric Psychiatry, 13, 795-801.

Márquez-González, M., Losada, A., Izal, M., PérezRojo, G. y Montorio, I. (2007). Modification of dysfunctional thoughts about caregiving in dementia family caregivers: Description and outcomes of an intervention programme. Aging and Mental Health, 11, 616-625.

Martín, M., Salvadó, I., Nadal, S., Miji, L.C., Rico, J.M., Lanz, P. et al. (1996). Adaptación para nuestro medio de la Escala de Sobrecarga del Cuidador (Caregiver Burden Interview) de Zarit. Revista de Gerontología, 6, 338-346.

Martínez-González, M.A., Sánchez- Villegas, A. y Faulin, J. (2006). Bioestadística amigable. (2 Edición). Madrid: Díaz de Santos.

Maydeu-Olivares, A., Rodríguez-Fornells, A., Gómez-Benito, J. y D'Zurilla, T.J. (2000). Psychometric properties of the Spanish adapta- 
tion of the Social Problem-Solving InventoryRevised (SPSI-R). Personality and Individual Differences, 29, 699-708.

McClendon, M.J., Smyth, K.A. y Neundorfer, M.M. (2004). Survival of persons with Alzheimer's disease: Caregiver coping matters. Gerontologist, 44, 508-519.

Mittelman, M.S., Roth, D.L., Coon, D.W., y Haley, W. E. (2004). Sustained benefit of supportive intervention for depressive symptoms in caregivers of patients with Alzheimer's disease. American Journal of Psychiatry, 161, 850- 856.

Montorio, I., Yanguas, J. y Díaz-Veiga, P. (1999). El cuidado del anciano en el ámbito familiar. En M. Izal y I. Montorio (Eds.), Gerontología conductual. Bases para la intervención y ámbitos de aplicación (pp. 141-180). Madrid: Síntesis.

Moral Serrano, M.S., Ortega, J.J., López Matoses, M.J. y Pellicer Magraner, P. (2003). Profile and risk of mental illness in caregivers for home care patients. Atención Primaria, 32, 77-87.

Mossialos, E., Allin, S. y Figueras, J. (Eds.). (2007). Health Systems in Transition: Template for analysis. Copenhagen: WHO Regional Office for Europe on behalf of the European Observatory on Health Systems and Policies. Obtenido el 31 de marzo de 2008 de http://www.euro.who.int/ Document/E88699.pdf

Muñoz Adanes, A. (2005). Does quantity generate quality? Testing the fundamental principle of brainstorming. Spanish Journal of Psychology, 8, 215-220.

Muñoz, R.F., Le, H.N., Clarke, G. y Jacox, L. (2002). Preventing the onset of major depression. En I.H. Gotlieb y C.L. Hammen (Eds.), Handbook of depression (pp. 343-359). New York: Guildford.

Nezu, A.M., Nezu, C.M. y Perri, M.G. (1989). Problem-solving therapy for depression. Theory, research, and clinical guidelines. New York: John Wiley \& Sons.

Organización Mundial de la Salud (2004). Prevention and treatment of mental disorders. Effective interventions and policy options. Summary report. Ginebra: World Health Organization. Obtenido el 16 de Noviembre de 2008, de http://www.who.int/mental_health/evi- dencelen/prevention_of_mental_disorders_sr.pdf

Pinquart, M. y Sörensen, S. (2006). Helping caregivers of persons with dementia: Which interventions work and how large are their effects? International Psychogeriatrics, 18, 577-595.

Radloff, L.S. (1977). The CES-D Scale: A selfreport depression scale for research in the general population. Applied Psychological Measurement, 1, 385-401.

Rivera, J. (2001). Redes familiares en el cuidado del anciano con demencia. Análisis evolutivo de un estudio poblacional. Madrid: Consejo Económico y Social. Comunidad de Madrid.

Roberts, J., Browne, G., Milne, C., Spooner, L., Gafni, A., Drummond-Young, M., et al. (1999). Problem-solving counselling for caregivers of the cognitively impaired: Effective for whom? Nursing Research, 48, 162-172.

Schulz, R. y Beach, S.R. (1999). Caregiving as a risk factor for mortality: The caregiver health effects study. The Journal of the American Medical Association, 282, 2215-2219.

Schulz, R., O’Brien, A.T., Bookwala, J. y Fleissner, K. (1995). Psychiatric and physical morbidity effects of dementia caregiving: Prevalence, correlates and causes. Gerontologist, 35, 771-791.

Schmidt, G.L., Bonjean, M.J., Widem, A.C., Schefft, B.K. y Steele, D.J. (1988). Brief psychotherapy for caregivers of demented relatives: Comparison of two therapeutic strategies. Clinical Gerontologist, 7, 109-125.

Sörensen, S., Duberstein, P., Gill, D. y Pinquart, M. (2006). Dementia care: Mental health effects, interventions strategies, and clinical implications. Lancet Neurology, 5, 961-973.

Teri, L., Logsdon, R.G., Uomoto, J. y McCurry, S.M. (1997). Behavioral treatment of depression in dementia patients: A controlled clinical trial. The Journal of Gerontology, 52B, 159-166.

Vázquez, F.L. (2002). La técnica de la solución de problemas aplicada a la depresión mayor. Psicothema, 14, 516-522.

Vázquez, F.L., Blanco, V. y López, M. (2007). An adaptation of the Center for Epidemiologic Studies Depression Scale for use in non-psychiatric Spanish populations. Psychiatry Research, 149, 247-252. 
Vázquez, F.L. y Torres, A. (2007). Análisis sobre la investigación de la prevención de episodios nuevos de depresión. Clínica y Salud, 18, 221-246.

Vitaliano, P.P., Young, H.M. y Zhang, J. (2004). Is caregiving a risk factor for illness? Current Directions in Psychological Science, 13, 13-16.

Zanetti, O., Metitieri, T., Bianchetti, A. y Trabucchi, M. (1988). Effectiveness of an educational program for demented person's relatives. Archives of
Gerontology and Geriatrics, 6, 531-538.

Zarit, S.H., Reever, K.E. y Bach-Peterson, J. (1980). Subjetive burden of husbands and wives as caregivers: A longitudinal study. Gerontologist, 26, 260-266.

Zarit, S.H., Zarit, J.M. y Reever, K.E. (1982). Memory training for severe memory loss: Effects on senile dementia patients and their families. Gerontologist, 22, 373-377.

Artículo recibido: 07/04/2009

Revisión recibida: 02/06/2009

Aceptado: 02/07/2009 